\title{
Endothelial nitric oxide synthase gene polymorphisms and risk of diabetic nephropathy: a systematic review and meta-analysis
}

Bruno Schmidt Dellamea ${ }^{1 *}$, Lana Catani Ferreira Pinto ${ }^{1}$, Cristiane Bauermann Leitão ${ }^{1}$, Katia Gonçalves Santos ${ }^{2}$ and Luis Henrique Santos Canani ${ }^{1}$

\begin{abstract}
Background: Nitric oxide (NO) has numerous functions in the kidney, including control of renal and glomerular hemodynamics, by interfering at multiple pathological and physiologically critical steps of nephron function. Endothelial NOS (eNOS) gene has been considered a potential candidate gene to diabetic nephropathy (DN) susceptibility. Endothelial nitric oxide synthase gene (eNOS-3) polymorphisms have been associated with DN, however some studies do not confirm this association. The analyzed polymorphisms were 4b/4a, T-786C, and G986T.

Methods: The Preferred Reporting Items for Systematic Reviews and Meta-Analysis (PRISMA) statement was used in this report. Case-control studies that had diabetic patients with DN as cases and diabetic patients without nephropathy as controls, as well as that evaluated at least one of the three polymorphisms of interest were considered eligible. All studies published up until December 31 ${ }^{\text {st }}, 2012$ were identified by searching electronic databases. Hardy-Weinberg equilibrium assessment was performed. Gene-disease association was measured using odds ratio estimation based on the following genetic contrast/models: (1) allele contrast; (2) additive model; (3) recessive model; (4) dominant model and (4) co-dominant model.

Results: Twenty-two studies were eligible for meta-analysis (4b/a: 15 studies, T-786C: 5 studies, and G984T: 12 studies). Considering 4b/a polymorphism, an association with DN was observed for all genetic models: allele contrast $(\mathrm{OR}=1.14, \mathrm{Cl}: 1.04-1.25)$; additive $(\mathrm{OR}=1.77, \mathrm{Cl}: 1.37-2.28)$; recessive $(\mathrm{OR}=1.77, \mathrm{Cl}: 1.38-2,27)$; dominant $(\mathrm{OR}=1.12$, Cl: 1.01-1.24), with the exception for co-dominance model. As well, T-786C polymorphism showed association with all models, with exception for co-dominance model: allele contrast ( $\mathrm{OR}=1.22$, Cl: 1.07-1.39), additive $(\mathrm{OR}=1.52, \mathrm{Cl}: 1.18-1.97)$, recessive $(\mathrm{OR}=1.50, \mathrm{Cl}: 1.16-1.93)$, and dominant $(\mathrm{OR}=1.11, \mathrm{Cl}: 1.01-1.23)$. For the G894T polymorphism, an association with DN was observed in allelic contrast ( $O R=1.12, \mathrm{Cl}: 1.03-1.25)$ and co-dominance models (OR $=1.13, \mathrm{Cl}: 1.04-1.37)$.

Conclusions: In the present study, there was association of DN with eNOS 4b/a and T-786C polymorphism, which held in all genetic models tested, except for co-dominance model. G894T polymorphism was associated with DN only in allele contrast and in co-dominance model. This data suggested that the eNOS gene could play a role in the development of DN.
\end{abstract}

\footnotetext{
* Correspondence: brunodellamea@gmail.com

${ }^{1}$ Endocrine Division, Hospital de Clínicas de Porto Alegre, Ramiro Barcelos 2350, CEP 90035-903 Porto Alegre, RS, Brazil

Full list of author information is available at the end of the article
} 


\section{Background}

Nitric oxide (NO) is a short-lived gaseous lipophilic molecule produced in almost all tissues and organs [1,2]. It is a free radical that exerts a variety of biological actions under both physiological and pathological conditions [3]. NO is formed from its precursor L-arginine by a family of $\mathrm{NO}$ synthases (NOSs). NOS system consists of three distinct isoforms, encoded by three distinct genes, including neuronal (nNOS or NOS-1), inducible (iNOS or NOS-2), and endothelial (eNOS or NOS-3). The gene encoding eNOS is located on chromosome 7 (7q35-q36) and contains 26 exons, with an entire length of $21 \mathrm{~kb}[3,4]$.

NO has numerous functions in the kidney, including control of renal and glomerular hemodynamics, by interfering at multiple pathological and physiologically critical steps of nephron function. NO dilates both the afferent and the efferent arteriole, augmenting the glomerular filtration rate (GFR) and influencing renal sodium handling [5]. NO also mediates pressure natriuresis, maintenance of medullary perfusion, decrease of tubuloglomerular reabsorption, and modulation of renal sympathetic nerve activity [6]. The net effect of NO in the kidney is to promote natriuresis and diuresis, along with renal adaptation to dietary salt intake $[7,8]$.

eNOS gene has been considered a potential candidate gene to diabetic nephropathy (DN) susceptibility. Since 1998, several polymorphisms of the eNOS gene have been identified, and their association with various diseases has been explored. Three polymorphisms have been the subject of research in relation to $\mathrm{DN}$, however the results are highly variable. The polymorphisms potentially associated with DN are a 27-bp repeat in intron 4 (VNTR), the T-786C single nucleotide polymorphism (SNP) in the promoter region (rs2070744), and G894T missense mutation in exon 7 (rs1799983) [9]. Some of these polymorphisms are associated with reduction of either eNOS activity $(-786 \mathrm{C}$ in the promoter area) or plasma concentrations of $\mathrm{NO}$ (four repeats in intron 4) [2].

However, the potential association of eNOS gene variants with the induction and progression of DN remains controversial. Some authors found a higher frequency of eNOS polymorphisms in patients with end-stage renal disease (ESRD) and DN [10-17], but not all studies reported this association [18-20].

The objective of the present study was to evaluate if eNOS gene polymorphisms are associated with $\mathrm{DN}$ through a systematic review of the literature and a meta-analysis.

\section{Results and discussion}

Three-hundred and nine studies were identified, and 281 were excluded based on review of titles and abstracts (70 animal experimental studies, 17 pharmacological studies, 86 without adequate cases or controls, 58 without the genes or polymorphisms of interest, 3 review articles,
5 meta-analysis, 35 studies with multiple publications of the same data presented with different titles, 7 no accesses to original data even after contacting authors). Twenty-eight articles were eligible and had the full text evaluated. Six studies were excluded due to lack of information regarding genotypic distribution. A total of 22 studies fulfilled the eligible criteria and were included for the meta-analysis (Figure 1).

Clinical characteristics of individual studies are described in Table 1. Regarding quality assessment, the phenotype definitions as cases or controls were appropriated, but none of the studies included information if genotyping was performed by personnel blinded to clinical status. Of the 22 studies included, 15 provided 4054/3405 cases/ controls for 4b/a; 5 provided 1436/1286 cases/controls for T-786C; and 12 provided 3316/2765 cases/controls for G894T. The allelic frequency of 4b, T-786, and G894 in cases/controls was 6647/5702, 1863/1795, and 4691/4017 respectively (Table 2).

Hardy-Weinberg equilibrium (HWE) was assessed using exact test and P-value $<0.05$ were considered significant. Only 4 studies ( 1 study for T-786C; 2 for G984T; and 1 for $4 \mathrm{~b} / \mathrm{a}$ ) with controls were not in HWE (Table 2). These studies were subjected to a sensitive analysis, and their exclusion did not show significant difference on OR.

For the $4 \mathrm{~b} / \mathrm{a}$ polymorphism, an association with $\mathrm{DN}$ in all genetic models, except for co-dominance, was observed: allele contrast $(\mathrm{OR}=1.15, \mathrm{CI}(95 \%)$ : 1.05-1.25, $\left.\mathrm{P}_{\mathrm{Q}}<0.01, \mathrm{I}^{2}=66 \%\right)$; additive ( $\mathrm{OR}=1.52$, CI $(95 \%)$ : 1.18 1.97, $\left.\mathrm{P}_{\mathrm{Q}}<0.01, \mathrm{I}^{2}=62 \%\right)$; recessive $(\mathrm{OR}=1.50, \mathrm{CI}$ (95\%): 1.16-1.93, $\left.\mathrm{P}_{\mathrm{Q}}<0.01, \mathrm{I}^{2}=64 \%\right)$; and dominant $\left(\mathrm{OR}=1.11\right.$, CI $\left.(95 \%): 1.01-1.23, \mathrm{P}_{\mathrm{Q}}=0.01, \mathrm{I}^{2}=49 \%\right)$. Similarly, for the T-786C polymorphism the association with DN was found with all models, with exception for co-dominance model: allele contrast $(\mathrm{OR}=1.22$, CI (95\%): 1.07-1.39, $\left.\mathrm{P}_{\mathrm{Q}}=0.59, \mathrm{I}^{2}=0 \%\right)$, additive $(\mathrm{OR}=1.52, \mathrm{CI}(95 \%)$ : 1.18-1.97, $\left.\mathrm{P}_{\mathrm{Q}}<0.01, \mathrm{I}^{2}=62 \%\right)$, recessive $(\mathrm{OR}=1.50$, CI (95\%): 1.16-1.93, $\left.\mathrm{P}_{\mathrm{Q}}<0.01, \mathrm{I}^{2}=64 \%\right)$ and dominant $(\mathrm{OR}=$ 1.11, CI (95\%): 1.01-1.23, $\left.\mathrm{P}_{\mathrm{Q}}<0.01, \mathrm{I}^{2}=49 \%\right)$. The G894T polymorphism showed association with $\mathrm{DN}$ in allelic contrast $\left(\mathrm{OR}=1.12\right.$, CI (95\%): 1.03-1.25, $\left.\mathrm{P}_{\mathrm{Q}}<0.01, \mathrm{I}^{2}=75 \%\right)$ and co-dominance model ( $\mathrm{OR}=1.13$, CI (95\%): 1.04-1.37, $\mathrm{P}_{\mathrm{Q}}=0.01, \mathrm{I}^{2}=60 \%$ ) (Table 3 and Figure 2). A random model analysis was performed confirming the fixed model results.

Publication bias was observed for the majority of the polymorphisms evaluated and are presented as a funnel plot for $4 \mathrm{~b} / \mathrm{a}$ polymorphsism (Figure 3 ). In order to identify non published data, we performed manual search for abstracts in some of the major scientific meetings in the field in the last seven years. We estimated the effect of these potential publication biases using trim and fill method and no major differences were observed from the original results.

Since some studies included only subjects of specific ethnicities or with type 1 or type $2 \mathrm{DM}$, we performed a 


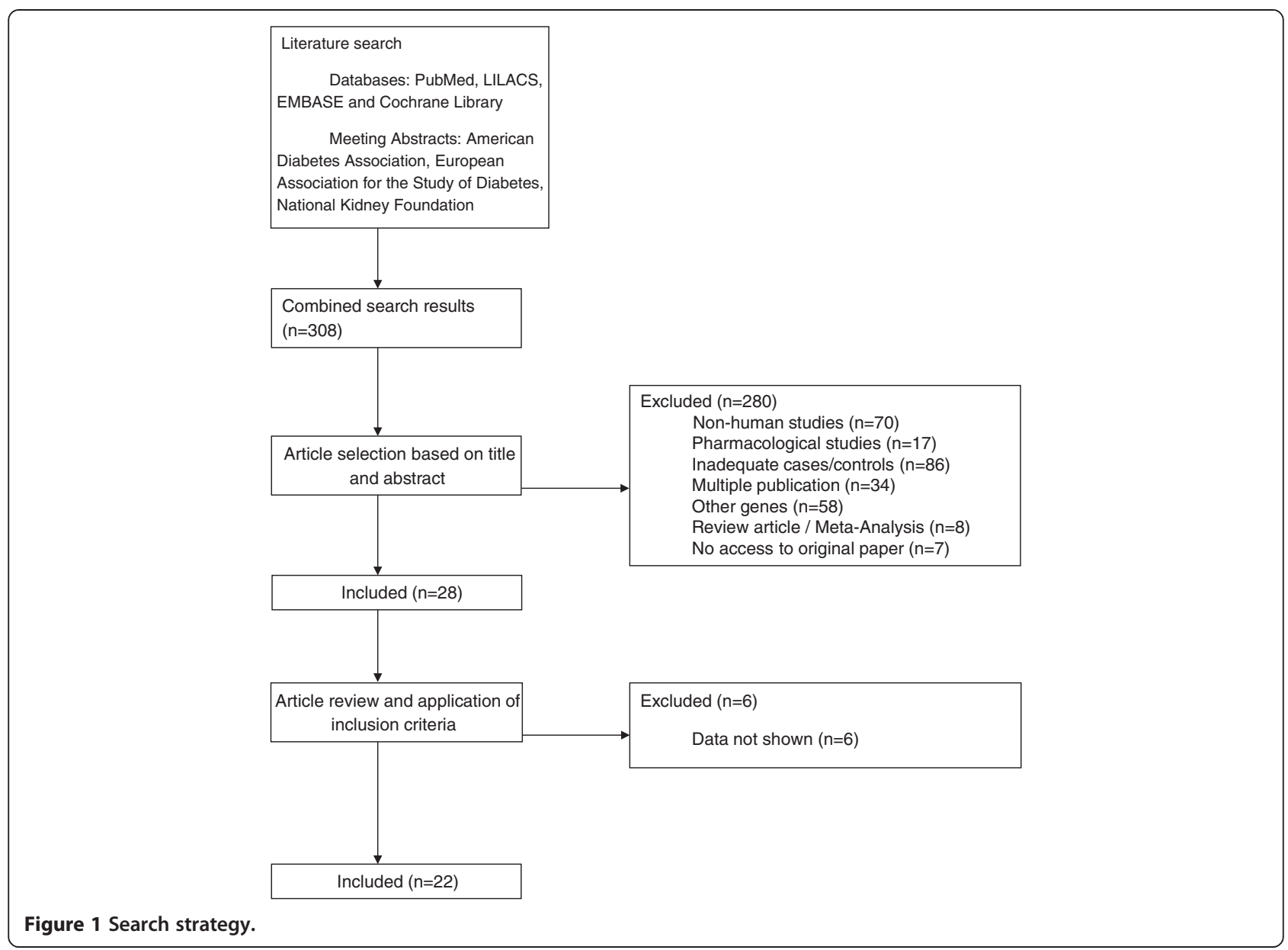

sensitive analysis stratifying the studies according to these characteristics. Considering 4b/a polymorphism, there was an association in White and East Asian populations in allele contrast, additive and recessive models; only for Whites in the dominant model; and none for the co-dominant model. For T-786C variant, no association was shown for Whites in allele contrast analysis or in any other genetic model, but in African populations the polymorphism was associated with $\mathrm{DN}$ in allele contrast, dominance and co-dominance models. Considering G894T polymorphism, in African populations the association was observed for all genetic models, with the exception of co-dominance model. There were insufficient studies to perform a meta-analysis for G894T in South Asians and West Asians.

According to the type of diabetes mellitus (DM), for $4 \mathrm{~b} / \mathrm{a}$ polymorphism an association was observed in additive and recessive models for both type 1 and type 2 diabetes, and only for type 1 in allele contrast and dominant models. There was no association with any type of DM in co-dominant model for $4 \mathrm{~b} / \mathrm{a}$ variant. For T-786C, no association in any genetic model was found in type 2 diabetes. There was insufficient data for this analysis in type $1 \mathrm{DM}$.
Likewise, for G894T variant there was an association only in the allele contrast model with type 2 diabetes (Table 3 ).

We compared the ORs of our meta-analysis with the results from a previous meta-analysis that used non-diabetic patients as controls [30]. The results were similar and no statistical differences in the ORs of the two studies were observed in all genetic models analyzed (data not shown).

\section{Conclusions}

In the present study, the most robust association of DN was with eNOS $4 \mathrm{~b} / \mathrm{a}$ and T-786C polymorphism that held in all genetic models tested, except for co-dominance model. G894T polymorphism was associated with DN only in allele contrast and in co-dominance model. 4b/ a polymorphism association with $\mathrm{DN}$ was confirmed in all ethnic groups evaluated and for all types of diabetes. The subgroup analysis of the T-786C variant should be viewed with caution, since it was limited due to the small number of studies.

Analyzing genetic model is important, considering the difference between them. Each individual genotype is formed by two alleles (for example G and T for G984T 
Table 1 Baseline studies characteristics

\begin{tabular}{|c|c|c|c|c|c|c|c|c|c|}
\hline Author & Year & Polymorphism & $\begin{array}{l}\text { Type } \\
\text { of DM }\end{array}$ & Ethnicity & Cases/controls (n) & Criteria & Male/female (\%) & Age & $\begin{array}{l}\text { DM duration } \\
\text { (years) }\end{array}$ \\
\hline \multirow[t]{2}{*}{ Ahluwalia et al. [21] } & 2008 & G894T, 4a/b, T-786C & 2 & East Asians & Case (195) & Overt proteinuria & $35 / 65$ & $60.0 \pm 6.15$ & $16.5 \pm 6.3$ \\
\hline & & & & & Control (255) & Normoalbuminuria & $41 / 59$ & $60.5 \pm 5.7$ & $15.6 \pm 5.2$ \\
\hline \multirow[t]{2}{*}{ Bessa et al. [22] } & 2011 & G894T & 2 & African & Case (40) & Albuminuria $>30 \mathrm{mg} / 24 \mathrm{~h}$ & $21 / 19$ & $58.8 \pm 12.5$ & $19.4 \pm 4.2$ \\
\hline & & & & & Control (40) & Albuminuria $<30 \mathrm{mg} / 24 \mathrm{~h}$ & $17 / 23$ & $55.4 \pm 8.8$ & $15.3 \pm 3.7$ \\
\hline \multirow[t]{2}{*}{ Cai et al. [23] } & 1998 & G894T & 2 & Whites & Case (116) & Microalbuminuria & NA & NA & NA \\
\hline & & & & & Control (284) & Normoalbuminuria & NA & NA & NA \\
\hline \multirow[t]{2}{*}{ Degen et al. [24] } & 2001 & $4 \mathrm{a} / \mathrm{b}$ & 1and 2 & Whites & Case (207) & AER $>30 \mathrm{mg} / 24 \mathrm{~h}$ & NA & NA & $>10 \mathrm{yrs}$ \\
\hline & & & & & Control (418) & AER $<30 \mathrm{mg} / 24 \mathrm{~h}$ & NA & NA & $>10 \mathrm{yrs}$ \\
\hline \multirow[t]{2}{*}{ Ezzidi et al. [10] } & 2008 & G894T, 4a/b, T-786C & 2 & African & Case (515) & AER $>30 \mathrm{mg} / 24 \mathrm{~h}$ & $46 / 54$ & $59.6 \pm 10.8$ & $13.5 \pm 6.3$ \\
\hline & & & & & Control (402) & AER $<30 \mathrm{mg} / 24 \mathrm{~h}$ & $42 / 58$ & $59.1 \pm 11.2$ & $11.5 \pm 6.2$ \\
\hline \multirow[t]{2}{*}{ Fujita et al. [25] } & 2000 & $4 a / b$ & 2 & East Asians & Case (102) & AER $>200 \mathrm{mcg} / \mathrm{min}$ & $60 / 40$ & $61.0 \pm 21.0$ & NA \\
\hline & & & & & Control (65) & AER $<20 \mathrm{mcg} / \mathrm{min}$ & $46 / 54$ & $62.0 \pm 10.0$ & NA \\
\hline \multirow[t]{2}{*}{ Ksiasek et al. [13] } & 2003 & $4 a / b$ & 2 & Whites & Case (178) & With DN & $48 / 52$ & $57.9 \pm 8.2$ & $8.7 \pm 3.1$ \\
\hline & & & & & Control (232) & Without DN & $51 / 49$ & $58.3 \pm 6.8$ & $8.0 \pm 2.6$ \\
\hline \multirow[t]{2}{*}{ Lin et al. [25] } & 2002 & $4 a / b$ & 2 & East Asians & Case (80) & With DN & NA & NA & NA \\
\hline & & & & & Control (48) & Normoalbuminuria & NA & NA & NA \\
\hline \multirow[t]{2}{*}{ Mollsten et al. [26] } & 2006 & G894T, 4a/b & 1 & Whites & Case (955) & AER $>20 \mathrm{mcg} / \mathrm{min}$ & $58 / 42$ & $40.3 \pm 10.0$ & $28(5-65)$ \\
\hline & & & & & Control (555) & AER $<20 \mathrm{mcg} / \mathrm{min}+\mathrm{DM}$ duration $>20 \mathrm{yrs}$ & $41 / 59$ & $42.2 \pm 10.2$ & $28(20-57)$ \\
\hline \multirow[t]{2}{*}{ Mollsten et al. [18] } & 2009 & G894T & 1 & Whites & Case (458) & AER $>300 \mathrm{mg} / 24 \mathrm{~h}$ & $39 / 61$ & $42.0 \pm 10.4$ & $27(7-65)$ \\
\hline & & & & & Control (319) & AER $<30 \mathrm{mg} / 24 \mathrm{~h}$ & $55 / 45$ & $43.7 \pm 11.0$ & $23(15-63)$ \\
\hline \multirow[t]{3}{*}{ Neuguebauer et al. [14] } & 2000 & $4 a / b$ & 2 & East Asians & Case 1 (104) & AER $20-200 \mathrm{mg} / \mathrm{g} \mathrm{Cr}$ & $53 / 47$ & $59.0 \pm 11.1$ & $13.8 \pm 5.1$ \\
\hline & & & & & Case 2 (39) & AER $>200 \mathrm{mg} / \mathrm{g} \mathrm{Cr}$ & $74 / 26$ & $59.0 \pm 8.6$ & $15.2 \pm 4.5$ \\
\hline & & & & & Control (82) & AER $<20 \mathrm{mg} / \mathrm{g} \mathrm{Cr}$ & $65 / 35$ & $56.0 \pm 8.6$ & $13.3 \pm 4.5$ \\
\hline \multirow[t]{3}{*}{ Rahimi et al. [27] } & 2012 & G894T & 2 & West Asians & Case 1 (68) & Albumin to creatinin ratio $>300 \mathrm{mg} / \mathrm{g}$ & $33 / 35$ & $57.1 \pm 8.7$ & $11.1 \pm 6.4$ \\
\hline & & & & & Case 2 (72) & Albumin to creatinin ratio $30-299 \mathrm{mg} / \mathrm{g}$ & $23 / 46$ & $55.3 \pm 8.6$ & $8.6 \pm 5.2$ \\
\hline & & & & & Control (72) & Albumin to creatinin ratio $<30 \mathrm{mg} / \mathrm{g}$ & $23 / 49$ & $54.4 \pm 7.9$ & $7.7 \pm 5.4$ \\
\hline \multirow[t]{2}{*}{ Rippin et al. [28] } & 2003 & $4 a / b$ & 1 & Whites & Case (464) & Overt proteinuria & NA & NA & NA \\
\hline & & & & & Control (396) & Normoalbuminuria & NA & NA & NA \\
\hline \multirow[t]{2}{*}{ Santos et al. [29] } & 2009 & G894T, 4a/b, T-786C & 2 & Whites & Case (376) & AER $>20 \mathrm{mcg} / \mathrm{min}$ or $>17 \mathrm{mg} / \mathrm{dl}$ & $57 / 43$ & $60.4 \pm 9.7$ & $15.0 \pm 9.1$ \\
\hline & & & & & Control (268) & AER $<20 \mathrm{mcg} / \mathrm{min}$ or $<17 \mathrm{mg} / \mathrm{dl}$ & $37 / 63$ & $62.0 \pm 9.4$ & $16.7 \pm 6.8$ \\
\hline \multirow[t]{2}{*}{ Shestakova et al. [16] } & 2006 & $4 a / b$ & 1 & Whites & Case (63) & AER $>300 \mathrm{mg} / 24 \mathrm{~h}$ & $47 / 53$ & $25.7 \pm 6.4$ & $12.6 \pm 2.8$ \\
\hline & & & & & Control (66) & AER $<30 \mathrm{mg} / 24 \mathrm{~h}$ & $37 / 63$ & $40.8 \pm 10.2$ & $26.8 \pm 6.9$ \\
\hline
\end{tabular}


Table 1 Baseline studies characteristics (Continued)

\begin{tabular}{|c|c|c|c|c|c|c|c|c|c|}
\hline \multirow[t]{3}{*}{ Shimizu et al. [30] } & 2002 & $4 a / b$ & 2 & East Asian & Case 1 (107) & Overt proteinuria & $70 / 30$ & $63.1 \pm 10.6$ & $15.5 \pm 11.0$ \\
\hline & & & & & Case 2 (124) & Overt proteinuria $+\mathrm{Cr}>1.5 \mathrm{mg} / \mathrm{dl}$ & $75 / 25$ & $65.1 \pm 8.8$ & $19.8 \pm 7.8$ \\
\hline & & & & & Control (203) & Normoalbuminuria $>\mathrm{DM}>10 \mathrm{yrs}$ & $65 / 35$ & $63.7 \pm 8.8$ & $18.6 \pm 7.8$ \\
\hline \multirow[t]{3}{*}{ Shin Shin et al. [31] } & 2004 & G894T & 2 & East Asians & Case 1 (35) & Microalbuminuria & $46 / 54$ & $62.9 \pm 10.9$ & $16(12-20)$ \\
\hline & & & & & Case 2 (83) & Overt proteinuria & $46 / 54$ & $58.8 \pm 9.7$ & $16(11-20)$ \\
\hline & & & & & Control (59) & Normoalbuminuric & $25 / 75$ & $61.6 \pm 11.7$ & $12(10-16)$ \\
\hline \multirow[t]{2}{*}{ Shoukry et al. [32] } & 2012 & G894T, 4a/b, T-786C & 2 & African & Case & Albumin to creatinin ratio $>300 \mathrm{mg} / \mathrm{g}$ & $108 / 92$ & $55.3 \pm 5.8$ & $14.5 \pm 4.3$ \\
\hline & & & & & Control & Albumin to creatinin ratio $<30 \mathrm{mg} / \mathrm{g}$ & $116 / 84$ & $54.6 \pm 5.2$ & $13.8 \pm 3.2$ \\
\hline \multirow[t]{2}{*}{ Tamemoto et al. [33] } & 2008 & G894T & NA & East Asians & Case (124) & Microalbuminuria & NA & NA & NA \\
\hline & & & & & Control (211) & Normoalbuminuria & NA & NA & NA \\
\hline \multirow[t]{4}{*}{ Taniwaki et al. [20] } & 2001 & $4 a / b$ & 2 & East Asians & Case 1 (44) & Microalbuminuria & $59 / 41$ & $60.5 \pm 8.5$ & $10.9 \pm 7.4$ \\
\hline & & & & & Case 2 (22) & Overt proteinuria & $68 / 32$ & $59.0 \pm 10.5$ & $12.8 \pm 6.5$ \\
\hline & & & & & Case 3 (20) & Overt proteinuria $+\mathrm{Cr}>1.5 \mathrm{mg} / \mathrm{dl}$ & $50 / 50$ & $64.2 \pm 7.8$ & $19.1 \pm 9.7$ \\
\hline & & & & & Control (69) & Normoalbuminuria & $59 / 41$ & $60.1 \pm 9.8$ & $7.4 \pm 4.5$ \\
\hline \multirow[t]{4}{*}{ Tiwari et al. [19] } & 2009 & G894T & 2 & South Asians & Case 1 (90) & $\mathrm{DM}>2 \mathrm{yrs}+\mathrm{Cr}>2 \mathrm{mg} / \mathrm{dl}$ from $\mathrm{N}$ India & $87 / 13$ & $53.6 \pm 11.0$ & $9.6 \pm 6.8$ \\
\hline & & & & & Case 2 (106) & $\mathrm{DM}>2 \mathrm{yrs}+\mathrm{Cr}>2 \mathrm{mg} / \mathrm{dl}$ from $\mathrm{S}$ India & $76 / 24$ & $55.9 \pm 11.5$ & $14.0 \pm 6.4$ \\
\hline & & & & & Control 1 (75) & $\mathrm{DM}>10 \mathrm{yrs}+\mathrm{Cr}<2 \mathrm{mg} / \mathrm{dl}$ from $\mathrm{N}$ India & $53 / 47$ & $61.0 \pm 8.9$ & $15.4 \pm 8.1$ \\
\hline & & & & & Control 2 (149) & $\mathrm{DM}>10 \mathrm{yrs}+\mathrm{Cr}<2 \mathrm{mg} / \mathrm{dl}$ from $\mathrm{S}$ India & $68 / 32$ & $60.5 \pm 11.4$ & $15.5 \pm 6.91$ \\
\hline \multirow[t]{3}{*}{ Zanchi et al. [17] } & 2000 & $4 a / b, T-786 C$ & 1 & Whites & Case 1 (74) & AER $>200 \mathrm{mcg} / \mathrm{mg}$ & $42 / 58$ & $35.5 \pm 7.3$ & $24.9 \pm 9.0$ \\
\hline & & & & & Case 2 (78) & AER $>200 \mathrm{mcg} / \mathrm{mg}+\mathrm{Cr}>1.5 \mathrm{mg} / \mathrm{dl}$ & $49 / 51$ & $35.7 \pm 6.5$ & $24.5 \pm 6.8$ \\
\hline & & & & & Control (195) & $\mathrm{AER}<20 \mathrm{mcg} / \mathrm{mg}+\mathrm{DM}>15 \mathrm{yrs}$ & $52 / 48$ & $36.5 \pm 7.6$ & $23.7 \pm 6.3$ \\
\hline
\end{tabular}

Where: AER : albumin excretion rate; DM: diabetes mellitus; DN: diabetic nephropathy; NA: not available; Cr: creatinine. 
Table 2 Polymorphisms distribution

\begin{tabular}{|c|c|c|c|c|c|c|c|}
\hline \multirow[t]{3}{*}{ Author } & \multicolumn{6}{|c|}{ Distribution of the T-786C polymorphism } & \multirow{3}{*}{$\begin{array}{c}\text { HWE } \\
\text { p value }\end{array}$} \\
\hline & \multicolumn{3}{|c|}{ Cases } & \multicolumn{3}{|c|}{ Controls } & \\
\hline & $\mathrm{TT}$ & $\mathrm{TC}$ & $\mathrm{CC}$ & $\mathrm{TT}$ & $\mathrm{TC}$ & $\mathrm{CC}$ & \\
\hline Ahluwalia et al. [21] & 121 & 62 & 12 & 165 & 87 & 3 & 0.020 \\
\hline Ezzidi et al. [10] & 261 & 215 & 34 & 224 & 139 & 32 & 0.115 \\
\hline Santos et al. 2011 [29] & 140 & 160 & 76 & 93 & 104 & 44 & 0.138 \\
\hline Shoukry et al. 2012 [32] & 57 & 89 & 54 & 84 & 83 & 33 & 0.129 \\
\hline \multirow[t]{4}{*}{ Zanchi et al. [17] } & 57 & 65 & 30 & 75 & 100 & 20 & 0.123 \\
\hline & \multicolumn{6}{|c|}{ Distribution of the G894T polymorphism } & HWE \\
\hline & \multicolumn{3}{|c|}{ Cases } & \multicolumn{3}{|c|}{ Controls } & $p$ value \\
\hline & GG & GT & TT & GG & GT & $\pi$ & \\
\hline Ahluwalia et al. [21] & 82 & 81 & 32 & 125 & 105 & 25 & 0.658 \\
\hline Bessa et al. 2011 [22] & 10 & 18 & 12 & 17 & 19 & 4 & 1.000 \\
\hline Cai et al. [23] & 65 & 44 & 7 & 148 & 109 & 27 & 0.310 \\
\hline Ezzidi et al. [10] & 185 & 247 & 81 & 165 & 195 & 41 & 0.151 \\
\hline Mollsten et al. [34] & 492 & 365 & 89 & 268 & 232 & 51 & 0.919 \\
\hline Mollsten et al. [18] & 293 & 133 & 32 & 182 & 121 & 16 & 0.540 \\
\hline Rahimi et al. 2012 [27] & 68 & 45 & 13 & 39 & 17 & 7 & 0.038 \\
\hline Santos et al. 2011 [29] & 176 & 166 & 32 & 118 & 95 & 22 & 0.640 \\
\hline Shin Shin et al. [31] & 95 & 23 & 0 & 52 & 7 & 0 & 1.000 \\
\hline Shoukry et al. 2012 [32] & 66 & 94 & 40 & 99 & 77 & 24 & 0.140 \\
\hline Tamemoto et al. [33] & 104 & 18 & 2 & 181 & 27 & 3 & 0.117 \\
\hline \multirow[t]{4}{*}{ Tiwari et al. [19] } & 82 & 21 & 3 & 91 & 43 & 13 & 0.035 \\
\hline & \multicolumn{6}{|c|}{ Distribution of the $4 b / 4 a$ polymorphism } & HWE \\
\hline & \multicolumn{3}{|c|}{ Cases } & \multicolumn{3}{|c|}{ Controls } & $p$ value \\
\hline & bb & ba & aa & bb & ba & aa & \\
\hline Ahluwalia et al. [21] & 146 & 28 & 21 & 189 & 61 & 5 & 1.000 \\
\hline Degen et al. [24] & 229 & 94 & 4 & 297 & 105 & 9 & 1.000 \\
\hline Ezzidi et al. [10] & 314 & 162 & 29 & 234 & 143 & 21 & 1.000 \\
\hline Fujita et al. [25] & 81 & 21 & 0 & 55 & 10 & 0 & 1.000 \\
\hline Ksiasek et al. 2003 [13] & 105 & 58 & 15 & 147 & 66 & 19 & 0.007 \\
\hline Lin et al. [26] & 115 & 21 & 1 & 41 & 6 & 1 & 0.271 \\
\hline Mollsten et al. [34] & 656 & 248 & 39 & 389 & 145 & 19 & 0.220 \\
\hline Neugebauer et al. [14] & 101 & 26 & 6 & 71 & 10 & 1 & 0.351 \\
\hline Rippin et al. [28] & 344 & 108 & 12 & 297 & 90 & 9 & 0.519 \\
\hline Santos et al. 2011 [29] & 237 & 99 & 11 & 168 & 59 & 5 & 1.000 \\
\hline Shestakova et al. [16] & 14 & 48 & 1 & 34 & 31 & 1 & 0.052 \\
\hline Shimizu et al. [30] & 180 & 44 & 6 & 156 & 44 & 3 & 1.000 \\
\hline Shoukry et al. 2012 [32] & 124 & 64 & 12 & 131 & 60 & 9 & 0.502 \\
\hline Taniwaki et al. [20] & 63 & 21 & 2 & 50 & 19 & 0 & 0.340 \\
\hline Zanchi et al. [17] & 80 & 27 & 37 & 144 & 47 & 4 & 1.000 \\
\hline
\end{tabular}

HWE (Hardy-Weinberg equilibrium).

polymorphism), and the risk of every genotype depends on the number of variant allele copies carried, where one of which is thought to be associated with a disease (e.g., T), association studies will collect information on the numbers of diseased and disease-free subjects with each of the three genotypes (GG, GT, and TT). So we 
Table 3 Meta-analysis in all genetic models with all patients and subgroup analysis, in fixed-model analysis, presenting heterogeneity $\left(\mathrm{P}_{\mathrm{Q}}\right.$ and $\left.\mathrm{I}^{2}\right)$

\begin{tabular}{|c|c|c|c|c|c|c|c|}
\hline & Population & Studies & OR & IC (95\%) & $\mathbf{P}$ & $\mathrm{P}_{\mathrm{Q}}$ & $I^{2}(\%)$ \\
\hline \multicolumn{8}{|l|}{$4 \mathrm{~b} / \mathrm{a}$} \\
\hline \multirow[t]{6}{*}{ Allele contrast } & All & 15 & 1.15 & $1.05-1,25$ & $<0.01$ & $<0.01$ & 66 \\
\hline & African & 2 & 0,98 & $0.81-1.18$ & 0.88 & 0,25 & 22 \\
\hline & East Asians & 6 & 1.21 & $0.97-1.50 .8$ & 0.08 & 0.29 & 18 \\
\hline & Whites & 7 & 1.20 & $1.07-1.34$ & $<0.01$ & $<0.01$ & 80 \\
\hline & Type 1 & 5 & 1.17 & $1.02-1.34$ & 0.02 & 0.07 & 54 \\
\hline & Type 2 & 10 & 1.12 & $0.99-1.27$ & 0.07 & 0.28 & 18 \\
\hline \multirow[t]{6}{*}{ Additive } & All & 15 & 1.52 & $1.18-1.97$ & $<0.01$ & $<0.01$ & 62 \\
\hline & African & 2 & 1,13 & $0,69-1,81$ & 0.62 & 0.56 & 0 \\
\hline & East Asians & 6 & 3.25 & $1.58-6.68$ & $<0.01$ & 0.31 & 16 \\
\hline & Whites & 7 & 1.49 & $1.06-2.08$ & 0.01 & $<0.01$ & 74 \\
\hline & Type 1 & 5 & 2.21 & $1.50-3.25$ & $<0.01$ & $<0.01$ & 81 \\
\hline & Type 2 & 11 & 1.36 & $0.98-1.88$ & 0.06 & 0.08 & 41 \\
\hline \multirow[t]{6}{*}{ Recessive } & All & 15 & 1.50 & $1.16-1.93$ & $<0.01$ & $<0.01$ & 64 \\
\hline & Africans & 2 & 1.13 & $0.69-1.83$ & 0,61 & 0.83 & 0 \\
\hline & East Asians & 6 & 3.44 & $1.68-7.05$ & $<0.01$ & 0.28 & 21 \\
\hline & Whites & 7 & 1.43 & $1.03-1.99$ & 0.03 & $<0.01$ & 75 \\
\hline & Type 1 & 5 & 2.19 & $1.49-3.21$ & $<0.01$ & $<0.01$ & 81 \\
\hline & Type 2 & 11 & 1.49 & $1.07-2.07$ & 0.02 & 0.08 & 42 \\
\hline \multirow[t]{6}{*}{ Dominant } & All & 15 & 1.11 & $1.01-1.23$ & 0.03 & 0,01 & 49 \\
\hline & African & 2 & 0.94 & $0.75-1.18$ & 0.64 & 0.24 & 27 \\
\hline & East Asians & 6 & 1.04 & $0.81-1.34$ & 0.71 & 0.44 & 0 \\
\hline & Whites & 7 & 1.20 & $1.05-1.36$ & $<0.01$ & $<0.01$ & 67 \\
\hline & Type 1 & 5 & 1.22 & $1.04-1.43$ & 0.01 & $<0.01$ & 78 \\
\hline & Type 2 & 11 & 1.05 & $0.92-1.20$ & 0.44 & 0.62 & 0 \\
\hline \multirow[t]{6}{*}{ Codominant } & All & 15 & 0.98 & 0.88-1.09 & 0.81 & 0,02 & 46 \\
\hline & African & 2 & 1.09 & $0.87-1.38$ & 0.42 & 0.29 & 7 \\
\hline & East Asians & 6 & 1.17 & $0.90-1.55$ & 0.22 & 0.14 & 38 \\
\hline & Whites & 7 & 0.90 & $0.79-1.04$ & 0.16 & 0.04 & 54 \\
\hline & Type 1 & 5 & 0.94 & $0.80-1.11$ & 0.46 & 0.01 & 68 \\
\hline & Type 2 & 11 & 1.01 & $0.88-1.17$ & 0.80 & 0.19 & 26 \\
\hline \multicolumn{8}{|l|}{ T-786C } \\
\hline \multirow[t]{4}{*}{ Allele contrast } & All & 5 & 1.28 & $1.14-1.44$ & $<0.01$ & 0.25 & 24 \\
\hline & African & 2 & 1.44 & $1.21-1.71$ & $<0.01$ & 0.26 & 19 \\
\hline & Whites & 2 & 1.13 & $0.94-1.36$ & 0.19 & 0.44 & 0 \\
\hline & Type 2 & 4 & 1.29 & $1.13-1.46$ & $<0.01$ & 0.15 & 42 \\
\hline \multirow[t]{4}{*}{ Additive } & All & 5 & 1.48 & $1.14-1.92$ & $<0,01$ & 0.01 & 67 \\
\hline & African & 2 & 1.43 & $0.98-2.09$ & 0.05 & 0.01 & 84 \\
\hline & Whites & 2 & 1.36 & 0.93-1.98 & 0.10 & 0.18 & 42 \\
\hline & Type 2 & 4 & 1.40 & $1.06-1.86$ & 0.01 & $<0.01$ & 73 \\
\hline \multirow[t]{3}{*}{ Recessive } & All & 5 & 1.38 & $1,09-1.76$ & $<0,01$ & 0.01 & 68 \\
\hline & African & 2 & 1.24 & $0.88-1.76$ & 0.21 & 0.01 & 81 \\
\hline & Whites & 2 & 1.39 & 0.98-1.95 & 0.06 & 0.09 & 0 \\
\hline
\end{tabular}


Table 3 Meta-analysis in all genetic models with all patients and subgroup analysis, in fixed-model analysis, presenting heterogeneity $\left(\mathbf{P}_{\mathbf{Q}}\right.$ and $\left.\mathrm{I}^{2}\right)$ (Continued)

\begin{tabular}{|c|c|c|c|c|c|c|c|}
\hline & Type 2 & 4 & 1.27 & $0.98-1.65$ & 0.06 & 0.01 & 72 \\
\hline \multirow[t]{4}{*}{ Dominant } & All & 5 & 1.21 & $1,04-1.42$ & 0.01 & 0.29 & 18 \\
\hline & African & 2 & 1.39 & $1.11-1.73$ & $<0.01$ & 0.13 & 54 \\
\hline & Whites & 2 & 1.05 & $0.81-1.37$ & 0.70 & 0.95 & 0 \\
\hline & Type 2 & 4 & 1.24 & $1.05-1.47$ & $<0.01$ & 0.22 & 31 \\
\hline \multirow[t]{4}{*}{ Codominant } & All & 5 & 0.95 & $0.81-1.11$ & 0.53 & 0.12 & 45 \\
\hline & African & 2 & 0.78 & $0.62-0.98$ & 0.03 & 0.48 & 0 \\
\hline & Whites & 2 & 1.15 & $0.89-1.49$ & 0.28 & 0.24 & 25 \\
\hline & Type 2 & 3 & 0.90 & $0.75-1.06$ & 0.20 & 0.131 & 15 \\
\hline \multicolumn{8}{|l|}{ G986T } \\
\hline \multirow[t]{6}{*}{ Allele contrast } & All & 12 & 1.12 & $1.03-1.21$ & $<0.01$ & $<0.01$ & 75 \\
\hline & African & 3 & 1.63 & $1.39-1.91$ & $<0.01$ & 0.61 & 0 \\
\hline & East Asian & 3 & 1.33 & $1.05-1.70$ & 0.01 & 0.74 & 0 \\
\hline & Whites & 4 & 0.93 & $0.84-1.04$ & 0.20 & 0.67 & 0 \\
\hline & Type 1 & 2 & 0.92 & $0.80-1.04$ & 0.18 & 0.18 & 0 \\
\hline & Type 2 & 9 & 1.27 & $1.15-1.42$ & $<0.01$ & $<0.01$ & 72 \\
\hline \multirow[t]{6}{*}{ Additive } & All & 12 & 1.19 & $0.99-1.43$ & 0.05 & $<0.01$ & 63 \\
\hline & African & 3 & 2.01 & $1.50-2.94$ & $<0.01$ & 0.27 & 22 \\
\hline & East Asian & 3 & 1.85 & $1,05-3.25$ & 0.03 & 0.59 & 0 \\
\hline & Whites & 4 & 0.86 & $0.67-1.10$ & 0.23 & 0.69 & 0 \\
\hline & Type 1 & 2 & 0.87 & $0.65-1.16$ & 0.34 & 0.44 & 0 \\
\hline & Type 2 & 9 & 1.47 & $1.16-1.86$ & $<0.01$ & $<0.01$ & 63 \\
\hline \multirow[t]{6}{*}{ Recessive } & All & 12 & 1.16 & $0.97-1.38$ & 0.09 & 0.02 & 52 \\
\hline & Africa & 3 & 1.80 & $1.31-2.46$ & $<0.01$ & 0.43 & 0 \\
\hline & East Asian & 3 & 1.73 & $1.01-2.96$ & 0.04 & 0.63 & 0 \\
\hline & Whites & 4 & 0.88 & $0.69-1.11$ & 0.29 & 0.62 & 0 \\
\hline & Type 1 & 2 & 0.91 & $0.69-1.20$ & 0.49 & 0.31 & 0 \\
\hline & Type 2 & 9 & 1.36 & $1.08-1.70$ & $<0.01$ & 0.03 & 53 \\
\hline \multirow[t]{6}{*}{ Dominant } & All & 12 & 0.99 & 0.89-1.11 & 0.92 & 0.07 & 45 \\
\hline & African & 3 & 1.46 & $1.17-1.82$ & $<0.01$ & 0.11 & 54 \\
\hline & East Asian & 3 & 1.32 & 0.98-1.79 & 0.06 & 0.73 & 0 \\
\hline & Whites & 4 & 0.93 & $0.80-1.07$ & 0.31 & 0.59 & 0 \\
\hline & Type 1 & 2 & 0.89 & $0.75-1.06$ & 0.19 & 0.74 & 0 \\
\hline & Type 2 & 9 & 1.19 & $0.92-1.26$ & 0.35 & 0.04 & 57 \\
\hline \multirow[t]{6}{*}{ Codominant } & All & 12 & 1.03 & $1.04-1.37$ & 0.01 & 0.01 & 60 \\
\hline & African & 3 & 0.92 & $0.74-1.14$ & 0.45 & 0.29 & 18 \\
\hline & East Asian & 3 & 0.89 & $0.65-1.21$ & 0.48 & 0.52 & 0 \\
\hline & Whites & 4 & 1.02 & $0.89-1.18$ & 0.69 & 0.44 & 0 \\
\hline & Type 1 & 2 & 1.08 & $0.91-1.29$ & 0.35 & 0.32 & 0 \\
\hline & Type 2 & 9 & 0.94 & $0.82-1.08$ & 0.41 & 0.34 & 11 \\
\hline
\end{tabular}

used the allele contrast, which compares the number of alleles $\mathrm{G}$ with the number of alleles $\mathrm{G}$; the additive model, which contrasts extreme homozygotes, comparing the genotype GG with the genotype TT; in recessive model two copies of $\mathrm{T}$ allele are essential to modify the risk, combining the GG and GT genotypes and comparing with TT; the dominant model, which heterozygous GT and homozygous TT genotypes have the similar risk as a single 
copy of $\mathrm{T}$ is sufficient to alter the risk, then compares GG with combined GT and TT genotypes; and the codominance model, commonly used genetic model, where each genotype gives a diverse and non additive risk. which combines the GG and TT genotypes and compares with GT. So OR in each particular genetic 


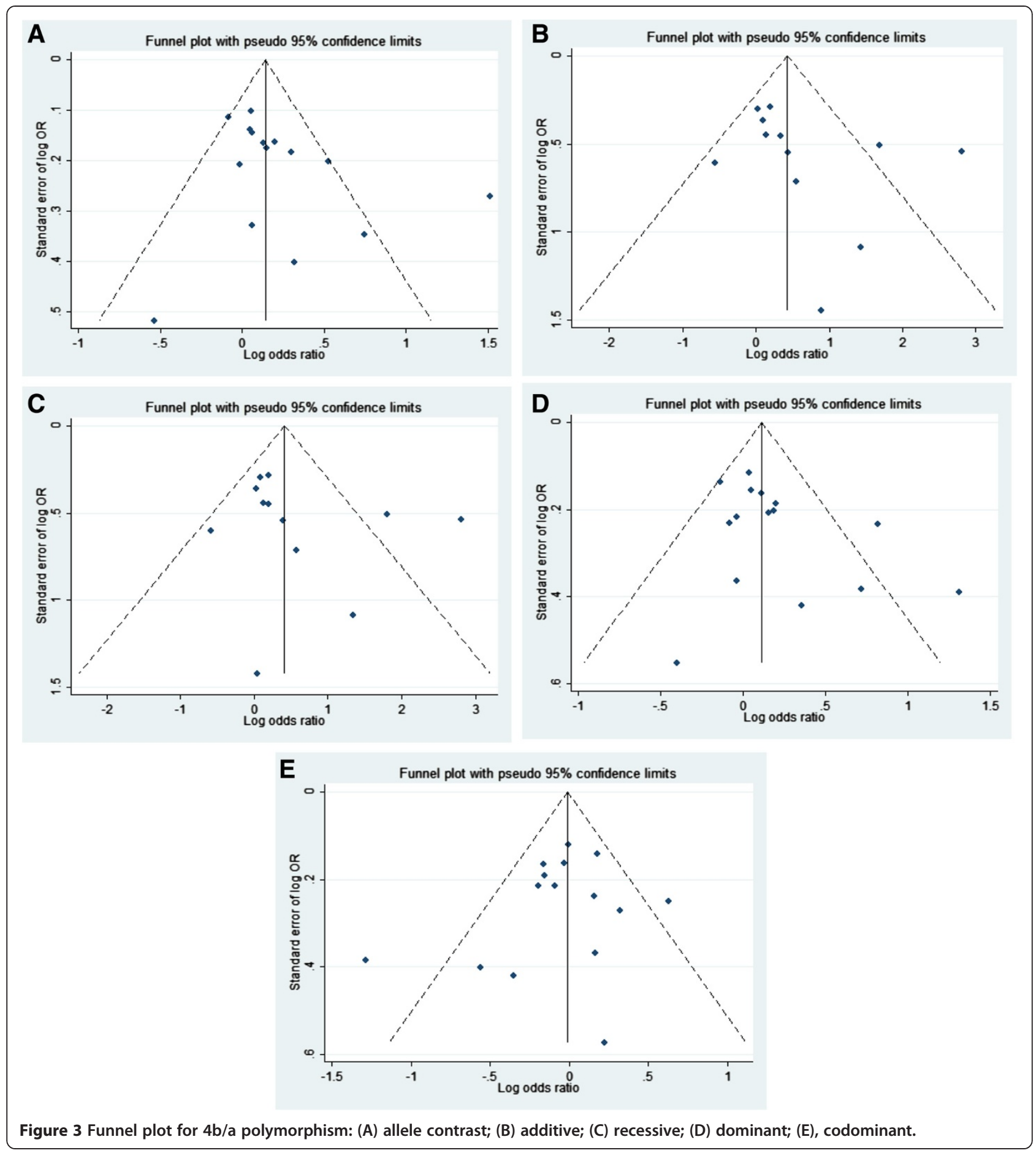

model gives us different interpretations about the risk of the polymorphisms.

These results are original and help to understand the role of these polymorphisms in the development of DN. However, it was not possible to exclude a publication bias of negative studies. Therefore, the exact effect could be smaller. As discussed before, other explanations, besides classic risk factors, are needed for understanding the progression of a diabetic patient from normoalbuminuria to macroalbuminuria, and a polymorphism identification of a specific gene would propitiate the development of a new therapy aimed directly to it.

In contrast to a recent meta-analysis performed by Zintzaras et al. [30], which analyzed the same polymorphisms in the progression of $\mathrm{DN}$, our analysis compared diabetic patients with DN (cases) with diabetic 
patients without DN (controls). In Zintzaras' study, healthy subjects were used as controls, mixed with patients with $\mathrm{DN}$. When the controls are defined as non-diabetic subjects, the observed association could reflect a genetic predisposition for individuals to develop "diabetic nephropathy". The obtained results could reflect a mixture of a susceptibility to diabetes per se and to nephropathy, which cannot be discriminated. In this regard, to serve non-diabetic individuals as controls seem rationale to estimate a risk of diabetic nephropathy. However, from clinical points of view, most of medical staff would be interested in risks for nephropathy among individuals with diabetes, as in the case with hyperglycemia, rather than combined risks for developing diabetes and for nephropathy thereafter. That is why diabetic individuals showing no or little nephropathy despite a term of duration have been widely investigated as controls, in most of the previous studies. So, our work and Zintzaras are derived from different standing points: a clinical aspect and a bio-mathematic research.

In this sense, we considered that the optimal control group when studding a DM complication is a diabetic patient without the complication and with disease duration long enough the permit a genetic predisposition to become clinically detected in the presence of hyperglycemia. Moreover, the disease duration must be comparable between cases and controls. Most included studies fulfilled the two pre-requisitions. As can be seen in Table 1, the DM duration is similar between cases and controls in each study and the majority has more than 10 years of DM, reflecting that authors from original studies probably took this important issue in consideration.

Despite the different control used by Zintzaras, they found 92 articles, being 20 included for meta-analysis; that provided 1942/1461 cases/controls for G894T, 2663/2232; cases/controls for $4 \mathrm{~b} / \mathrm{a}$, and $857 / 845$ cases/controls for T-786C. That was similar to ours that had 22 studies included, but provided about one third more cases/ controls. The OR observed in their analyzes showed significance in allelic contrast model for G894 polymorphism, recessive and additive model for $4 \mathrm{~b} / \mathrm{a}$ polymorphism, and allelic contrast model for T-786C, all observed in our study; but our analyze showed association in more genetic models than that, like codominant model for G894T; allele contrast and dominant model for $4 \mathrm{~b} / \mathrm{a}$; recessive, dominant and additive model for T786C. Furthermore, we compared our ORs with those reported by Zintzaras et al. and no statistical differences were found. With that said, our study reinforce the findings from Zintzaras.

DN development predisposition has not been fully explained, since glycemic control and environmental factors, as well as traditional risk factors, do not accurately predict the occurrence of this diabetic complication in all patients. With this in mind, studies have been trying to resolve this question using genetic approaches. Many candidate genes have been explored in this context, and eNOS polymorphisms have been implicated in the susceptibility to glomerular disease, by mechanisms yet unknown [15]. However, there is no consensus on the role of these polymorphisms in modulation of risk for $\mathrm{DN}$, since the available literature demonstrates mixed results and most of the studies have a small sample. In this scenario, the recommended approach to help investigators in understanding the effect of each polymorphism in DN development is a systematic review and meta-analysis. Our data suggest an association between eNOS polymorphisms and DN. Assuming a recessive model, the relative risk, attributable risk and population attributable risk for the $4 \mathrm{a}$ variant ranges are, respectively, $1.20 ; 0.11$; and 0.09 .

The present paper has some limitations. The inclusion of studies evaluating patients with DM in several stages of $\mathrm{DN}$, ranging from microalbuminuria to chronic renal insufficiency in kidney replacement therapy, could bias the results due to clinical heterogeneity of cases. Some studies did not present the data separated by DN stages. Furthermore, inclusion criteria in the reviewed studies utilized different methods and cutoffs to define microalbuminuria or macroalbuminuria. Although all clinically validated [35], these aspects made impossible to evaluate the effect of each polymorphism in the stages of DN in this meta-analysis. Finally, the polymorphisms true effects could be overestimated in the present study, since there is some indication of publication bias.

In conclusion, this study shows an association between $\mathrm{DN}$ and polymorphisms in eNOS gene. This effect is very consistent for the $4 \mathrm{~b}$ and $\mathrm{T}-786$ polymorphism.

\section{Methods}

The Preferred Reporting Items for Systematic Reviews and Meta-Analysis (PRISMA) statement was used in this report [36,37].

\section{Selection criteria and search strategy}

Case-control studies that had diabetic patients with DN as cases and diabetic patients without nephropathy as controls, as well as that evaluated at least one of the three polymorphisms of interest (4b/4a, T-786C, G986T) were considered eligible. Only studies in humans and using validated genotyping methods were considered. No publication language, publication date, or publication status restrictions were imposed. All studies published up until December $31^{\text {st }}, 2012$ were identified by searching electronic databases: Medline (1966-Present), EMBASE (1980-Present), LILACS and Cochrane Library.

Abstracts presented at scientific events held by: The American Diabetes Association (ADA); The European Association for the Study of Diabetes (EASD); The National Kidney Association (NKA); and The American Society 
of Nephrology (ASN) were searched over the last seven years. The authors were contacted for more details in the case of abstracts with missing information.

The following index terms were used: ("Nitric Oxide Synthase Type III" OR "NOS3 protein, human") AND ("Databases, Genetic" OR "Genetic Predisposition to Disease" OR "Genetic Phenomena" OR "Genetic Processes" OR "Genetic Markers" OR "Genetic Variation" OR "Polymorphism, Genetic" OR "Genetic Research" OR "Genetic Determinism" OR "Genes" OR "Genetics" OR "Mutation" OR "Genetics, Medical" OR "DNA") AND ("Proteinuria" OR "Albuminuria" OR "Kidney Failure" OR "Kidney Failure, Chronic" OR "Kidney Diseases" OR "Diabetic Nephropathies").

\section{Study selection and data extraction}

Eligibility assessment was made by title and abstracts review and in doubtful cases by full article review. This was performed independently in a standardized manner by two investigators (BSD and CBL). Disagreements between reviewers were resolved by consensus.

Two investigators extracted the data, one independent to another (BSD and LCFP). Disagreements were resolved by a third author (LHC). For articles with missing information, $(\mathrm{n}=3)$ the authors were contacted for further information, but none responded. In the case of duplicate publications, the first manuscript published was included in the analysis. Information was extracted from each individual study based on: (1) characteristics of study participants (including age, gender, type of diabetes, diabetes duration, nephrologic status, and ethnicity) [38], (2) case and control definition; (3) genetic data (including allelic distribution and genotypic frequency).

\section{Quality assessment}

To ascertain the validity of each eligible case-control study, two investigators (BSD and LCFP) worked independently during the initial search and after worked together to determine the adequacy of studies selection. It was assessed if the same exclusion criteria for cases and controls were used; if cases were easily differentiated from controls; if analysis of studied polymorphisms were conducted in a standard, valid, and reliable way, if major biases were identified and considered in design and analysis; and how good the study was to minimize the risks of bias or confusion. Hardy-Weinberg equilibrium assessment among the control group within each polymorphism in all studies was checked by exact test using an online HWE calculator (http://ihg.gsf.de/cgi-bin/hw/hwa1.pl).

\section{Statistical analysis}

Gene-disease association was measured using odds ratio estimation based on the following genetic contrast/models: (1) allele contrast; (2) additive model; (3) recessive model;
(4) dominant model and (4) co-dominant model [39,40]. Heterogeneity was tested by chi-squared test, Cochran's Q, and inconsistency with $\mathrm{I}^{2}$. If $\mathrm{P}_{\mathrm{Q}}<0.10$, then heterogeneity was considered statistically significant. Odds ratio was calculated using fixed-effect models (Mantel-Haenszel), and random models when heterogeneity was observed. Multiple comparisons were not made because meta-analysis of genetic association studies is considered an exploratory study, without a prespecified key hypothesis [41,42].

The risk of publication bias was evaluated using funnel plot graphics [43].

Sensibility tests were made concerning to ethnia and type of diabetes.

Data were analyzed using Stata/SE 11.2 (http://www. stata.com).

We compared the ORs of our meta-analysis with the results from a previous one [44] that used non-diabetic patients as controls using the differences of OR and 95\% CI (WinPepi version 11.3).

\section{Abbreviations}

ADA: The American Diabetes Association; ASN: The American Society of Nephrology; DM: Diabetes mellitus; DN: Diabetic nephropathy; EASD: The European Association for the Study of Diabetes; eNOS: Endothelial nitric oxide synthase; ESRD: End stage renal disease; GFR: Glomerular filtration rate; iNOS: Inducible nitric oxide synthase; NKA: The National Kidney Association; nNOS: Neuronal nitric oxide synthase; NO: Nitric oxide; NOS: Nitric oxide synthase; PRISMA: The preferred reporting items for systematic reviews and meta-analysis; SNP: Single nucleotide polymorphism.

\section{Competing interests}

The authors declare that they have no competing interest.

\section{Authors' contributions}

BSD participated in design, selection of included articles, data collection, statistical analysis and wrote the manuscript; LCFP participated in data collection; CBL participated in selection of included articles and wrote the manuscript; KGS data collection and wrote the manuscript, LHSC wrote the manuscript. All authors read and approved the final manuscript.

\section{Funding}

LHC and CBL received a scholarship from Conselho Nacional de Desenvolvimento Cinetífico e Tecnológico (CNPq).

\section{Author details}

${ }^{1}$ Endocrine Division, Hospital de Clínicas de Porto Alegre, Ramiro Barcelos 2350, CEP 90035-903 Porto Alegre, RS, Brazil. ²Universidade Luterana do Brasil, Canoas, Brazil.

Received: 25 July 2012 Accepted: 6 January 2014

Published: 16 January 2014

\section{References}

1. Moncada S, Palmer RM, Higgs EA: Nitric oxide: physiology, pathophysiology, and pharmacology. Pharmacol Rev 1991, 43(2):109-142.

2. Asakimori $Y$, et al: $T(-786)->C$ polymorphism of the endothelial nitric oxide synthase gene influences the progression of renal disease. Nephron 2002, 91(4):747-751.

3. Bredt DS, Snyder SH: Nitric oxide: a physiologic messenger molecule. Annu Rev Biochem 1994, 63:175-195.

4. Tsutsui $M$, et al: Nitric oxide synthases and cardiovascular diseases: insights from genetically modified mice. Circ J 2009, 73(6):986-993.

5. Zoccali C: The endothelium as a target in renal diseases. J Nephro/ 2007, 20(Suppl 12):S39-S44. 
6. Mount PF, Power DA: Nitric oxide in the kidney: functions and regulation of synthesis. Acta Physiol (Oxf) 2006, 187(4):433-446.

7. Lahera $V$, et al: Effects of NG-nitro-L-arginine methyl ester on renal function and blood pressure. Am J Physiol 1991, 261(6 Pt 2):F1033-F1037.

8. Shultz PJ, Tolins JP: Adaptation to increased dietary salt intake in the rat. Role of endogenous nitric oxide. J Clin Invest 1993, 91(2):642-650.

9. Liu Y, et al: T-786C polymorphism of the endothelial nitric oxide synthase gene is associated with albuminuria in the diabetes heart study. J Am Soc Nephrol 2005, 16(4):1085-1090.

10. Ezzidi l, et al: Association of endothelial nitric oxide synthase Glu298Asp, $4 \mathrm{~b} / \mathrm{a}$, and $-786 \mathrm{~T}>\mathrm{C}$ gene variants with diabetic nephropathy. J Diabetes Complications 2008, 22(5):331-338.

11. Bellini $\mathrm{MH}$, et al: Association of endothelial nitric oxide synthase gene intron 4 polymorphism with end-stage renal disease. Nephrology (Carlton) 2007, 12(3):289-293.

12. Buraczynska $M$, et al: Endothelial nitric oxide synthase gene intron 4 polymorphism in patients with end-stage renal disease. Nephrol Dial Transplant 2004, 19(9):2302-2306.

13. Ksiazek $P$, et al: Endothelial nitric oxide synthase gene intron 4 polymorphism in type 2 diabetes mellitus. Mol Diagn 2003, 7(2):119-123.

14. Neugebauer S, Baba T, Watanabe T: Association of the nitric oxide synthase gene polymorphism with an increased risk for progression to diabetic nephropathy in type 2 diabetes. Diabetes 2000, 49(3):500-503.

15. Mamoulakis D, et al: Intron 4 polymorphism of the endothelial nitric oxide synthase eNOS gene and early microangiopathy in type 1 diabetes. Int J Immunogenet 2009, 36(3):153-157.

16. Shestakova MV, et al: The relationship between genetic and haemodynamic factgors in diabetic nephropathy (DN): case-control study in type 1 diabetes mellitus (T1DM). Diabetes Res Clin Pract 2006, 74s:10.

17. Zanchi A, et al: Risk of advanced diabetic nephropathy in type 1 diabetes is associated with endothelial nitric oxide synthase gene polymorphism. Kidney Int 2000, 57(2):405-413.

18. Mollsten A, et al: The endothelial nitri oxide synthase gene and risk pf diabetic nephropathy and development of cardiovascular disease in type 1 diabetes. Mol Genet Metab 2009, 97:5.

19. Tiwari AK, et al: Oxidative stress pathway genes and chronic renal insufficiency in Asian Indians with Type 2 diabetes. J Diabetes Complications 2009, 23(2):102-111.

20. Taniwaki $\mathrm{H}$, et al: Relations between ACE gene and ecNOS gene polymorphisms and resistive index in type 2 diabetic patients with nephropathy. Diabetes Care 2001, 24(9):1653-1660.

21. Ahluwalia TS, et al: Endothelial nitric oxide synthase gene haplotypes and diabetic nephropathy among Asian Indians. Mol Cell Biochem 2008, 314(1-2):9-17.

22. El-Din Bessa SS, Hamdy SM: Impact of nitric oxide synthase Glu298Asp polymorphism on the development of end-stage renal disease in type 2 diabetic Egyptian patients. Ren Fail 2011, 33(9):878-884

23. Cai $H$, et al: A common Glu298->Asp (894G->T) mutation at exon 7 of the endothelial nitric oxide synthase gene and vascular complications in type 2 diabetes. Diabetes Care 1998, 21(12):2195-2196.

24. Degen B, Schmidt S, Ritz E: A polymorphism in the gene for the endothelial nitric oxide synthase and diabetic nephropathy. Nephrol Dial Transplant 2001, 16(1):185.

25. Fujita $\mathrm{H}$, et al: Lack of association between an ecNOS gene polymorphism and diabetic nephropathy in type 2 diabetic patients with proliferative diabetic retinopathy. Horm Metab Res 2000, 32(2):80-83.

26. Lin S, Qu H, Qiu M: Allele A in intron 4 of ecNOS gene will not increase the risk of diabetic nephropathy in type 2 diabetes of Chinese population. Nephron 2002, 91(4):768.

27. Rahimi Z, Vaisi-Raygani A, Parsian A: Concomitant presence of endothelial nitric oxide 894T and angiotensin II-converting enzyme D alleles are associated with diabetic nephropathy in a Kurdish population from Western Iran. Nephrology (Carlton) 2012, 17(2):175-181.

28. Rippin JD, et al: Nitric oxide synthase gene polymorphisms and diabetic nephropathy. Diabetologia 2003, 46(3):426-428.

29. Santos KG, et al: Association of eNOS gene polymorphisms with renal disease in Caucasians with type 2 diabetes. Diabetes Res Clin Pract 2011, 91(3):353-362.

30. Shimizu T, et al: Endothelial nitric oxide synthase gene and the development of diabetic nephropathy. Diabetes Res Clin Pract 2002, 58(3):179-185.
31. Shin Shin Y, et al: Relations between eNOS Glu298Asp polymorphism and progression of diabetic nephropathy. Diabetes Res Clin Pract 2004, 65(3):257-265.

32. Shoukry A, et al: Endothelial nitric oxide synthase gene polymorphisms and the risk of diabetic nephropathy in type 2 diabetes mellitus. Genet Test Mol Biomarkers 2012, 16(6):574-579.

33. Tamemoto H, Ishikawa SE, Kawakami M: Association of the Glu298Asp polymorphism of the eNOS Gene with ischemic heart disease in Japanese diabetic subjects. Diabetes Res Clin Pract 2008, 80(2):275-279.

34. Mollsten A, et al: Glu298Asp and NOS4ab polymorphisms in diabetic nephropathy. Ann Med 2006, 38(7):522-528.

35. Gross $\mathrm{L}$, et al: Diabetic nephropathy: diagnosis, prevention, and treatment. Diabetes Care 2005, 28(1):164-176.

36. Moher D, et al: Preferred reporting items for systematic reviews and meta-analyses: the PRISMA statement. Ann Intern Med 2009, 151(4):264-269. W64.

37. Liberati A, et al: The PRISMA statement for reporting systematic reviews and meta-analyses of studies that evaluate health care interventions: explanation and elaboration. Ann Intern Med 2009, 151(4):W65-W94.

38. Jorde LB, Wooding SP: Genetic variation, classification and 'race'. Nat Genet 2004, 36(11 Suppl):S28-S33.

39. Zintzaras E, Lau J: Synthesis of genetic association studies for pertinent gene-disease associations requires appropriate methodological and statistical approaches. J Clin Epidemio/ 2008, 61(7):634-645.

40. Minelli $C$, et al: The choice of a genetic model in the meta-analysis of molecular association studies. Int J Epidemiol 2005, 34(6):1319-1328.

41. Rothman KJ: No adjustments are needed for multiple comparisons. Epidemiology 1990, 1(1):43-46.

42. Bender R, Lange S: Adjusting for multiple testing-when and how? J Clin Epidemiol 2001, 54(4):343-349.

43. Egger $M$, et al: Bias in meta-analysis detected by a simple, graphical test BMJ 1997, 315(7109):629-634.

44. Zintzaras E, Papathanasiou AA, Stefanidis I: Endothelial nitric oxide synthase gene polymorphisms and diabetic nephropathy: a HuGE review and meta-analysis. Genet Med 2009, 11(10):695-706.

doi:10.1186/1471-2350-15-9

Cite this article as: Dellamea et al:: Endothelial nitric oxide synthase gene polymorphisms and risk of diabetic nephropathy: a systematic review and meta-analysis. BMC Medical Genetics 2014 15:9.

\section{Submit your next manuscript to BioMed Central and take full advantage of:}

- Convenient online submission

- Thorough peer review

- No space constraints or color figure charges

- Immediate publication on acceptance

- Inclusion in PubMed, CAS, Scopus and Google Scholar

- Research which is freely available for redistribution

Submit your manuscript at www.biomedcentral.com/submit
C) Biomed Central 